\title{
Comparison Study of 2D Images of Temperature Fluctuations during Sawtooth Oscillation with Theoretical Models
}

\author{
H. K. Park, ${ }^{1}$ A. J. H. Donné, ${ }^{2}$ N. C. Luhmann, Jr., ${ }^{3}$ I. G. J. Classen, ${ }^{2}$ C. W. Domier, ${ }^{3}$ E. Mazzucato, ${ }^{1}$ T. Munsat, ${ }^{4}$ \\ M. J. van de Pol, ${ }^{2}$ Z. Xia, ${ }^{2}$ and TEXTOR Team ${ }^{5}$ \\ ${ }^{1}$ Princeton Plasma Physics Laboratory, Princeton, New Jersey, USA \\ ${ }^{2}$ FOM-Institute for Plasma Physics Rijnhuizen*, Association EURATOM-FOM, P.O. Box 1207, 3430 BE Nieuwegein, The Netherlands \\ ${ }^{3}$ University of California at Davis, Davis, California, USA \\ ${ }^{4}$ University of Colorado at Boulder, Boulder, Colorado, USA \\ ${ }^{5}$ Forschungszentrum Jülich GmbH*, Institut für Plasmaphysik, Association EURATOM-FZJ, D-52425 Jülich, Germany \\ (Received 30 January 2006; published 19 May 2006)
}

\begin{abstract}
High temporal and spatial resolution two-dimensional (2D) images of electron temperature fluctuations were employed to study the sawtooth oscillation in the Toroidal Experiment for Technically Oriented Research tokamak plasmas. The 2D images are directly compared with the expected 2D patterns of the plasma pressure (or electron temperature) from various theoretical models. The observed experimental 2D images are only partially in agreement with the expected patterns from each model: The image of the initial reconnection process is similar to that of the ballooning mode model. The intermediate and final stages of the reconnection process resemble those of the full reconnection model. The time evolution of the images of the hot spot or island is partially consistent to those from the full reconnection model but is not consistent with those from the quasi-interchange model.
\end{abstract}

DOI: 10.1103/PhysRevLett.96.195004

PACS numbers: 52.35.Vd, 52.30.Cv, 52.55.Fa

The "sawtooth oscillation" was discovered in the early days of fusion plasma research [1] and is known as the $m / n=1 / 1$ internal kink mode, where $m$ and $n$ are poloidal and toroidal mode numbers, respectively. An excellent review of recent research in the field of sawtooth oscillations is given in Ref. [2]. This Letter presents a direct comparison study between experimentally measured highresolution 2D images of electron temperature fluctuations with the relevant $2 \mathrm{D}$ pattern from prominent physical models developed for the sawtooth oscillation physics in high temperature plasmas. The experiment was performed in the Toroidal Experiment for Technically Oriented Research (TEXTOR) tokamak plasma, which has a circular cross section, a major radius of $175 \mathrm{~cm}$, and a minor radius of $46 \mathrm{~cm}$ [3]. The toroidal magnetic field in the present work was in the range 1.9-2.4 T, and the corresponding plasma current was $<305 \mathrm{kA}$. Key plasma parameters were as follows: The central electron density and temperature are in the range $(1.5-2.5) \times 10^{19} \mathrm{~m}^{-3}$ and from 1.2 to $1.6 \mathrm{keV}$, respectively. The corresponding peak toroidal beta is $\sim 1.0 \%$, and the average poloidal beta is between 0.3 and 0.5 . The toroidal rotation of the plasma varied from $\sim 1$ to $8 \times 10^{4} \mathrm{~m} / \mathrm{s}$. The speed of a thermal electron is $\sim 6 \times 10^{7} \mathrm{~m} / \mathrm{s}$. The Alfvén and ion acoustic speeds are $5 \times 10^{6}$ and $7 \times 10^{5} \mathrm{~m} / \mathrm{s}$, respectively. Using plasma parameters close to the $q \sim 1$ surface, the characteristic reconnection time $\left(\tau_{c}\right)$ is $\sim 700 \mu \mathrm{s}$.

High-resolution 2D images of the electron temperature fluctuations in TEXTOR have been measured with a 2D electron cyclotron emission imaging (ECEI) system. The basic principle of the technique is similar to that of con- ventional 1D ECE radiometers $[4,5]$. The new feature of the ECEI diagnostic is that measurements are done in a 2D matrix of sample volumes. Since the ECEI diagnostic has recently been published elsewhere [6,7], we only briefly mention it here as an introduction to the comparison with theoretical models. The system has 16 (vertical) $\times$ 8 (horizontal) sampling volumes arranged in a $2 \mathrm{D}$ matrix of $16 \mathrm{~cm}$ (vertical) $\times 7 \mathrm{~cm}$ (radial). The vertical full width at half maximum of the central antenna pattern is $2 \mathrm{~cm}$ and slightly worsens at the top and bottom edges; the radial resolution is $\sim 1 \mathrm{~cm}$ across the core of the tokamak plasma (total 128 channels). The time resolution of the system is primarily limited by the digitizer, and the fastest time scale can be up to $5 \mu \mathrm{sec}$. Radial extensions of the image can be obtained with a variation of the LO source frequency and/or the applied magnetic field. The fluctuation quantities are relatively calibrated to the averaged value obtained with a long integration time, and the intensity of the images is represented by $\delta T_{e} /\left\langle T_{e}\right\rangle$, where $T_{e}$ is the electron temperature, \langle\rangle is the time average, $\delta T_{e}$ is the fluctuation level $\left(=T_{e}-\left\langle T_{e}\right\rangle\right)$, and $\left\langle T_{e}\right\rangle$ is constant for the duration of many sawtooth oscillations. The first measurements with the ECEI system on the reconnection process in TEXTOR have been recently published [8]. This Letter focuses on a direct comparison with three leading physical models developed for the sawtooth crash phenomenon.

In the full reconnection model $[9,10]$, the plasma current density in the core region increases $[q(0)$ drops below unity], and the $m / n=1 / 1$ internal kink mode becomes unstable due to a pressure driven instability. Island formation starts due to an influx of the cooler part of the plasma 
outside the inversion radius through the magnetic reconnection as soon as the pressure driven instability reconnects the magnetic field through the reconnection zone along the magnetic pitch of the $q \sim 1$ surface. As the island (the region with $q \sim 1$ ) grows, the hot spot (the region with $q<1$ ) gets smaller and it is eventually eliminated, and the island fully occupies the core on a reconnection time scale defined as $\tau_{c} \approx \frac{1}{2} \sqrt{\tau_{A}^{*} \tau_{\eta}}$, where $\tau_{A}^{*}$ is the modified Alfvén transit time and $\tau_{\eta}$ is the resistive diffusion time in Refs. [9,10]. Second, the quasi-interchange model [11] differs significantly from the full reconnection model and does not require any magnetic field reconnection process. The core plasma having a flat $q$ profile $(q \sim 1)$ inside the inversion radius becomes unstable due to a slight change of the magnetic pitch angle. In this model, there is no pressure driven instability. As the hot spot deforms into a crescent shape, the cooler outside portion of the plasma is convectively inducted into the core region, resulting in a flattening of the core pressure profile. This model was experimentally supported by x-ray tomography [12] on the Joint European Tokamak; however, a later experimental study [13] on the Tokamak de Varrenes concluded that the tomographic reconstruction is ill-posed and that there is no unique solution for the image through the inversion process of a limited number of chordal measurements from only a few independent views. The distinctively different evolution of the hot spot and/or cold island formation between the quasi-interchange model and full reconnection model could not be conclusively identified due to the lack of reliable 2D experimental tools. Furthermore, the observation that there is no significant change $[14,15]$ in the core plasma current $[q(0)$ was changed only from 0.7 to 0.8 and stayed below $\sim 1.0$ ] before and after the sawtooth crash is inconsistent with both models. Note that there exists an experimental result [16] that $q(0)$ was changed from $\sim 0.7$ before the reconnection to $\sim 1.0$ after reconnection in a shaped plasma. This discrepancy further motivated modeling of the driven reconnection process of the sawtooth oscillation as a secondary reconnection process [17]. Observation of a localized electron temperature bulge $[18,19]$ at the low field side on the poloidal plane in the Tokamak Fusion Test Reactor (TFTR) device has been interpreted as caused by a finite pressure effect on the sawtooth oscillation [20]. Here a steep pressure gradient near the temperature bulge at the low field side leads to a global stochasticity of the magnetic field which is thought to be necessary to reconcile the small change of the current density and the fast change of the pressure during the reconnection time observed in finite beta $[\beta=$ $\left.\left(2 \mu_{0} \sum_{j=i, e} n_{j} T_{j} / B^{2}\right)\right]$ plasmas. Finally, the pressure driven ballooning mode instability [21] was introduced to account for the observed disruptions lead by a sawtooth crash in the high beta $\left[\beta_{p} \sim 1\right.$ and $\left.\beta_{t}(0) \sim 4 \%\right]$ plasmas [18,19] in TFTR. These modes are more pronounced at the bad curvature side of the magnetic surface (low field side of the torus). Also, a 3D local reconnection model where the reconnection zone is localized in the toroidal plane with many assumptions has been proposed in Ref. [18]. In plasmas with a moderate beta $\left[\beta_{p} \sim 0.4\right.$ and $\beta_{t}(0) \sim$ $1 \%$, where the present $2 \mathrm{D}$ imaging measurements were conducted, the level of the ballooning modes and global stochasticity of magnetic field lines that are strongly coupled with the pressure surfaces is moderate compared to those at high beta plasmas as demonstrated in Ref. [19]. All models developed to explain the sawtooth oscillation are based on numerous assumptions, and thus there is a strong need to compare them with precise experimental results.

A representative view of the sequence of $2 \mathrm{D}$ ECEI images during the reconnection process is provided in Fig. 1. The time history of the temperature fluctuation measured by one of the 128 channels (innermost channel at $z=0$ ) illustrates the typical precursor oscillations as the plasma rotates in the toroidal direction. The corresponding
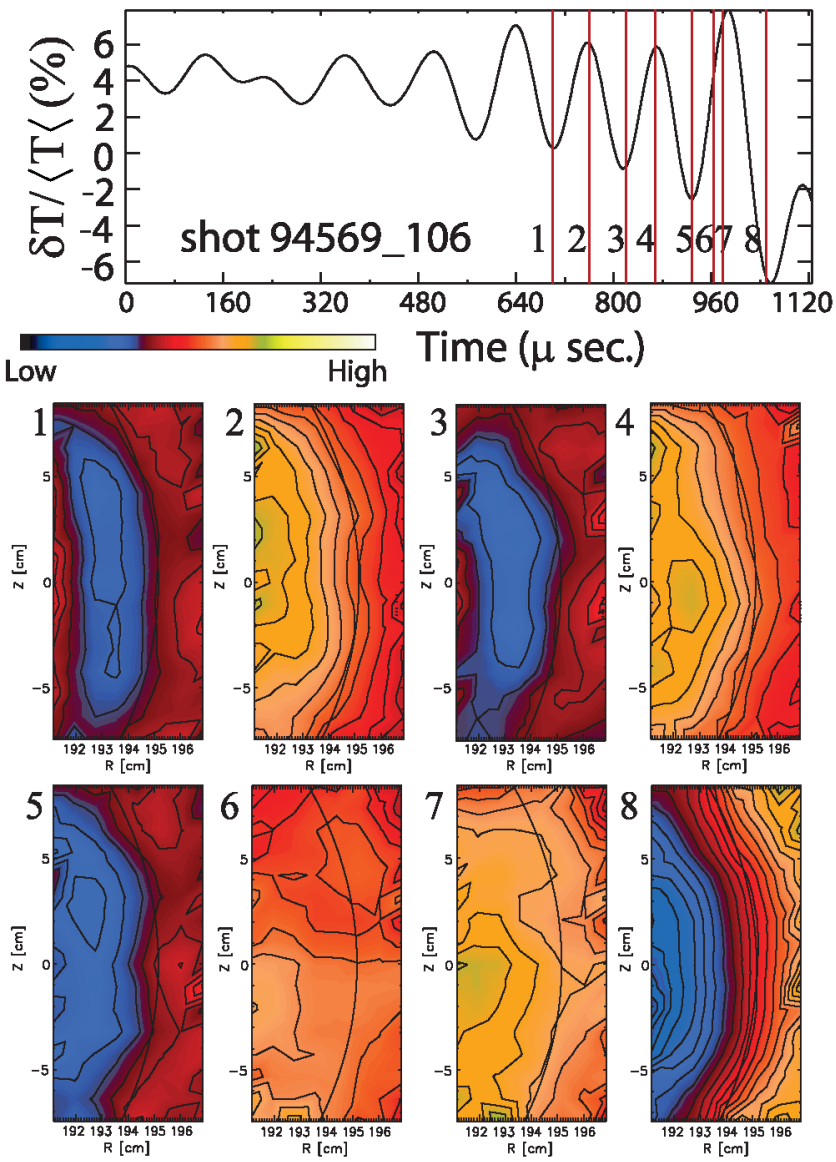

FIG. 1 (color). High-resolution single frame images of the time evolution of the hot spot or island (cold spot) while the plasma is rotating along the toroidal direction. During the precursor phase, the island is growing as the $m / n=1 / 1$ mode swells toward the low field side but does not show any sign of the heat flow until a sharp temperature point develops and heat crosses the inversion radius. 
images at each time slice are shown in the figure. The toroidal rotation is driven mainly by the momentum of the heating beam and the estimated speed is $\sim 8 \times 10^{4} \mathrm{~m} / \mathrm{s}$. Note that the phase of each reconnection process at the view position is strongly governed by the local nature of the reconnection event and the plasma rotation speed. In the precursor stage, the growth of island or hot spot is clearly illustrated. Before the heat flow crosses the inversion radius, distortion of the $m / n=1 / 1$ mode (sharp temperature point) in the bottom at the low field side is clearly illustrated in frame 6 after the symmetric images in Fig. 1. The sharp temperature point even crosses the inversion radius and leads to a puncture of the magnetic surface as the plasma rotates and the heat flow across the inversion radius ends at the top of the view as illustrated in frame 7 of this figure.

The evolution of the hot spot or island in the early stage of the precursor period is compared with the relevant images from the full reconnection model and the quasiinterchange model in Fig. 2. In the full reconnection model, the formation of the island is an indication of the topological change of the magnetic field through the reconnection at the low field side. One of the frames from the simulation results in Ref. [14] is directly compared with frames 4 (hot spot) and 5 (island) from Fig. 1 as shown in Fig. 2(a). The shape and growth of the island in Fig. 2(a) are strikingly similar to those from simulation results of the full reconnection model. On the other hand, the shape of the hot spot is circular and it swells as it approaches the crash time, whereas the hot spot in the model is shrinking as the island grows in simulation. In the experimental result, there is no indication of a heat flow until the reconnection through the sharp temperature point takes place. In the full reconnection model, the formation of the island is the beginning of the reconnection process, since it is assumed that the island is the result of a topological change of the magnetic field

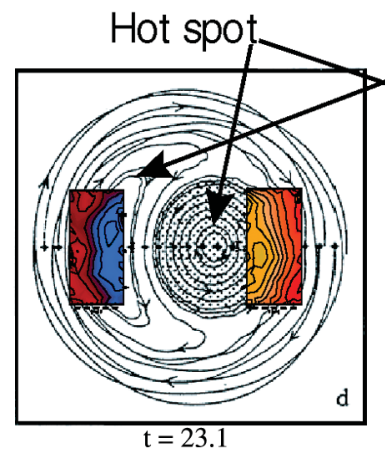

(a)

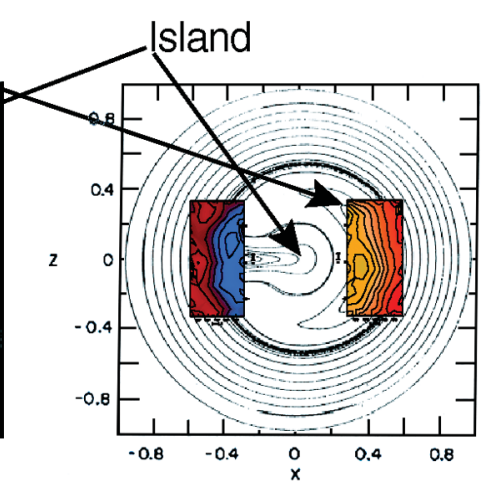

(b)
FIG. 2 (color). Experimental 2D images of the hot spot and island formation from Fig. 1 are directly overlaid for comparison on the 2D line patterns from (a) the full reconnection model and (b) the quasi-interchange model. structure. The reconnection time, estimated based on the definition of the characteristic time $\left(\tau_{c}\right)$ which starts with the time when island formation is observed (precursor) and ends with the full island formation in the core for this experimental condition, is $\sim 600 \mu \mathrm{s}$, which is consistent with the estimated value of $\tau_{c}$. However, it is notable that a trace of heat flow outside of the inversion radius was routinely observed in the later stage of the precursor as shown in Fig. 1. If the time when a trace of the heat flow outside of the inversion radius is detected is regarded as the beginning of the reconnection process, the reconnection time is less than $\sim 100 \mu \mathrm{s}$. This observation suggests a new physical mechanism which may delay the reconnection process until a critical time while the island grows. Alternatively, the reconnection process is based on two distinctive phases; the first phase is an extremely weak reconnection, while a stronger reconnection driven by a pressure mode follows in the second phase. Often, the "crash time" is referred to as the time period from the maximum value of $T_{e}(0)$ to the minimum value of $T_{e}(0)$ when there are no precursors, whereas the characteristic reconnection time $\left(\tau_{c}\right)$ is referred to as the time period from the moment when the island is formed during the precursor phase (indication of reconnection at the lower field side) during the precursor phase to the moment when the island is fully established.

In the quasi-interchange model, the hot spot deforms into a crescent shape due to a strong distortion of the magnetic surface of the $m / n=1 / 1$ mode that results from the lack of magnetic shear, and the cooler parts of the plasma are convectively induced to the concave side of the crescent shaped hot spot as shown in Fig. 2(b). Therefore, any magnetic field line reconnection process is not required to explain the sawtooth oscillation. It is rather clear that the time evolution of the island (cold spot) in the experimental 2D images is distinctively different from this model. Furthermore, the localized reconnection does occur with a sharp pressure point and heat flow crosses the inversion radius, whereas this model does not require any type of reconnection process. Since the occurrence frequency of the full reconnection type of the sawtooth crash is dominant, the pressure instability driven reconnection may be the dominant mechanism compared to the magnetic instability.

The sharp temperature point or "pressure finger" accompanied with the swelling of the $m / n=1 / 1$ mode at the low field side of the torus is the signature of the ballooning mode model [21,22]. Dispersion of the heat is dominated by the global stochastic magnetic field in this model. The magnitude of the pressure finger and the global stochasticity of the magnetic field are small at the moderate plasma beta. In Fig. 3, the observed 2D images of the reconnection processes on the poloidal plane are compared to those from the simulation results of the ballooning mode model [22] for a similar plasma beta $\left(\beta_{p}=0.4\right.$ and 


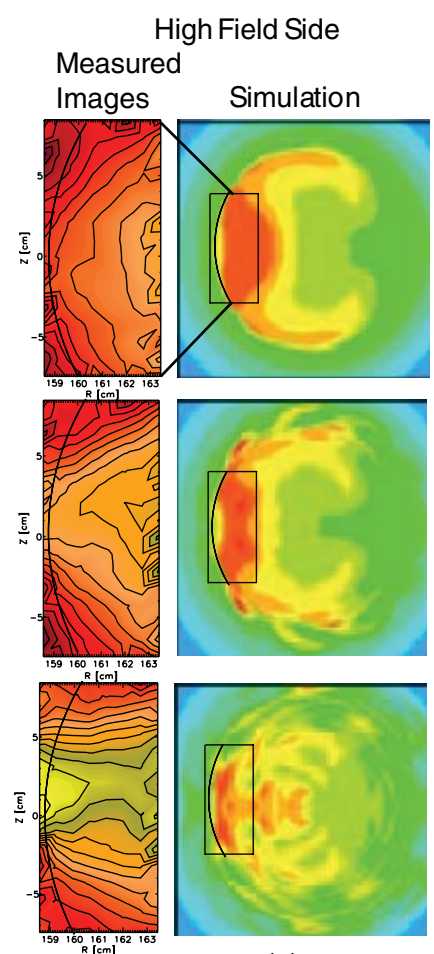

(a)

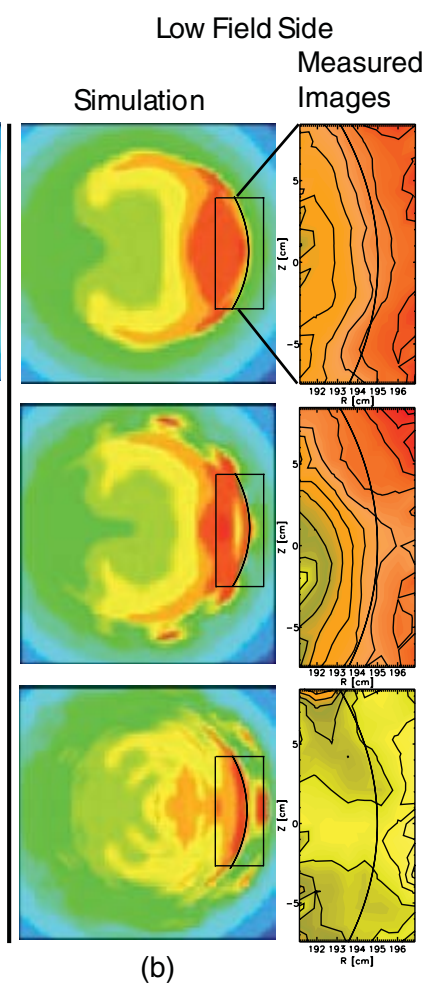

(b)
FIG. 3 (color). Three frames (before the ballooning mode is formed, with the ballooning mode, and stochastic pressure pattern after the ballooning mode) from the simulation in Ref. [22] are directly compared with the relevant 2D images. (a) High field side. (b) Low field side.

$\beta_{t} \sim 2 \%$ ). Three 2D images (before the presence of the ballooning mode, ballooning mode, and crash phase) are directly compared to the $2 \mathrm{D}$ pressure pattern of the ballooning mode in the bad curvature (low field) side from the simulation [22]. The pressure bulge with a smooth surface before the development of the ballooning mode is quite similar as shown in the top frame in Fig. 3(b). In the middle frame in Fig. 3(b), the sharp temperature point is strikingly similar to the ballooning mode from the simulation. While the stochastic behavior is dominant in the pressure pattern of the simulation, the experimentally measured heat flow patterns are highly collective as shown in the bottom of Fig. 3(b). At the good curvature side of the torus (high field side), the measured 2D image before development of the ballooning mode is quite similar as shown in the top frame in Fig. 3(a). In the middle frame in Fig. 3(a), instead of the pressure finger as shown in the low field side, the $m / n=$ $1 / 1$ mode is indented toward the center while the observed 2D image of the sharp temperature point resembles that of the low field side. Like the low field case, the global stochasticity of the pressure pattern is dominant in simu- lation while the heat flow is highly collective in the high field side [bottom of Fig. 3(a)].

The observed 2D images of the electron temperature fluctuations during the reconnection time are directly compared with characteristic 2D patterns from three leading physical models. The time evolution of the hot spot and island partly resembles that of the full reconnection model, but it is not consistent with those of the quasi-interchange model. A pressure driven instability (sharp temperature point due to the distortion) of the $m / n=1 / 1$ mode accompanied with a kink instability or pressure bulge due to a finite pressure effect on the $m=1$ mode is consistent with the ballooning mode model, but the fact that the observed heat transport in the poloidal plane is well organized (collective behavior) suggests that the global stochasticity of the magnetic field line is not the dominant mechanism for this case.

The authors are grateful to Dr. H. Soltwisch, Dr. W. Park, Dr. E. Fredrickson, and Dr. M. Yamada for valuable discussions. This work is supported by the U.S. DOE, Contracts No. DE-AC02-76-CH0-3073, No. DE-FG0395ER-54295, and No. W-7405-ENG-48, NWO, and EURATOM.

*Partners in the Trilateral Euregio Cluster.

[1] S. von Goeler et al., Phys. Rev. Lett. 33, 1201 (1974).

[2] R. J. Hastie, Astrophys. Space Sci. 256, 177 (1997).

[3] A. J. H. Donné et al., Fusion Sci. Technol. 47, 220 (2005).

[4] I. Hutchinson, Plasma Diagnostics (Cambridge University, New York, 1987).

[5] G. Bekefi, Radiation Processes in Plasmas (Wiley, New York, 1966).

[6] H. Park et al., Rev. Sci. Instrum. 75, 3787 (2004).

[7] H. Park et al., Rev. Sci. Instrum. 74, 4239 (2003).

[8] H. Park et al., Phys. Plasmas (to be published).

[9] B. B. Kadomtsev, Sov. J. Plasma Phys. 1, 389 (1975).

[10] A. Sykes and J.A. Wesson, Phys. Rev. Lett. 37, 140 (1976).

[11] J. A. Wesson, Plasma Phys. Controlled Fusion 28, 243 (1986).

[12] R. S. Granetz et al., Nucl. Fusion 28, 457 (1988).

[13] C. Janicki et al., Nucl. Fusion 30, 950 (1990).

[14] H. Soltwisch, Rev. Sci. Instrum. 59, 1599 (1988).

[15] F. M. Levinton et al., Phys. Fluids B 5, 2554 (1993).

[16] B. W. Rice et al., Rev. Sci. Instrum. 70, 815 (1999).

[17] D. Biskamp and J.F. Drake, Phys. Rev. Lett. 73, 971 (1994).

[18] Y. Nagayama et al., Phys. Plasmas 3, 1647 (1996).

[19] E. D. Fredrickson et al., Phys. Plasmas 7, 5051 (2000).

[20] W. Park et al., Phys. Fluids B 3, 507 (1991).

[21] W. Park et al., Phys. Rev. Lett. 75, 1763 (1995).

[22] Y. Nishimura et al., Phys. Plasmas 6, 4685 (1999). 